\title{
Real-time spatial frequency domain imaging by single snapshot multiple frequency demodulation technique
}

Zili Cao

Weihao Lin

Xinlin Chen

Bixin Zeng Follow this and additional works at: https://digitalcommons.fairfield.edu/physics-facultypubs

Dapyxight 2017 Society of Photo Optical Instrumentation Engineers (SPIE). One print or

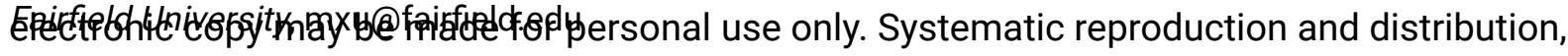
duplication of any material in this publication for a fee or for commercial purposes, or modification of the contents of the publication are prohibited.

The final publisher PDF has been archived here with permission from the copyright holder.

\section{Peer Reviewed}

\section{Repository Citation}

Cao, Zili; Lin, Weihao; Chen, Xinlin; Zeng, Bixin; and Xu, Min, "Real-time spatial frequency domain imaging by single snapshot multiple frequency demodulation technique" (2017). Physics Faculty Publications. 134.

https://digitalcommons.fairfield.edu/physics-facultypubs/134

\section{Published Citation}

Zili Cao, Weihao Lin, Xinlin Chen, Bixin Zeng, Min Xu, "Real-time spatial frequency domain imaging by single snapshot multiple frequency demodulation technique", Proc. SPIE 10059, Optical Tomography and Spectroscopy of Tissue XII, $100590 Z$ (17 February 2017); doi: 10.1117/12.2253992; https://doi.org/10.1117/12.2253992

This item has been accepted for inclusion in DigitalCommons@Fairfield by an authorized administrator of DigitalCommons@Fairfield. It is brought to you by DigitalCommons@Fairfield with permission from the rightsholder(s) and is protected by copyright and/or related rights. You are free to use this item in any way that is permitted by the copyright and related rights legislation that applies to your use. For other uses, you need to obtain permission from the rights-holder(s) directly, unless additional rights are indicated by a Creative Commons license in the record and/or on the work itself. For more information, please contact digitalcommons@fairfield.edu. 


\section{Real-time spatial frequency domain imaging by single snapshot multiple frequency demodulation technique}

Zili Cao, Weihao Lin, Xinlin Chen, Bixin Zeng, Min Xu

Zili Cao, Weihao Lin, Xinlin Chen, Bixin Zeng, Min Xu, "Real-time spatial frequency domain imaging by single snapshot multiple frequency demodulation technique," Proc. SPIE 10059, Optical Tomography and Spectroscopy of Tissue XII, $100590 Z$ (17 February 2017); doi: $10.1117 / 12.2253992$

SPIE. Event: SPIE BiOS, 2017, San Francisco, California, United States 


\title{
Real-time Spatial Frequency Domain Imaging by Single Snapshot Multiple Frequency Demodulation Technique Zili Cao ${ }^{\mathrm{a}}$, Weihao Lin ${ }^{\mathrm{a}}$, Xinlin Chen ${ }^{\mathrm{a}}$, Bixin Zeng ${ }^{\mathrm{a}}$, and Min Xu* \\ ${ }^{a}$ Insititute of Lasers and Biophotonics, Department of Biomedical Engineering, Wenzhou Medical University, Wenzhou 325035, China; ${ }^{b}$ Department of Physics, Fairfield University, Fairfield, Connecticut 06824, USA
}

\begin{abstract}
We have presented a novel Single Snapshot Multiple Frequency Demodulation (SSMD) method enabling single snapshot wide field imaging of optical properties of turbid media in the Spatial Frequency Domain. SSMD makes use of the orthogonality of harmonic functions and extracts the modulation transfer function (MTF) at multiple modulation frequencies and of arbitrary orientations and amplitudes simultaneously from a single structured-illuminated image at once. SSMD not only increases significantly the data acquisition speed and reduces motion artifacts but also exhibits excellent noise suppression in imaging as well. The performance of SSMD-SFDI is demonstrated with experiments on both tissue mimicking phantoms and in vivo for recovering optical properties. SSMD is ideal in the implementation of a real-time spatial frequency domain imaging platform, which will open up SFDI for vast applications in, for example, mapping the optical properties of a dynamic turbid medium or monitoring fast temporal evolutions.
\end{abstract}

Keywords: spatial frequency domain imaging, single snapshot multiple frequency demodulation (SSMD), real time, turbid medium, optical properties

\section{INTRODUCTION}

Spatial Frequency Domain Imaging (SFDI) is one emerging non-contact and quantitative wide field modality for rapid mapping of the optical properties of turbid media such as biological tissue [1,2]. It has attracted significant attention recently as SFDI has been proved effective in probing the micro-architecture of the sub-surface of the turbid medium over a wide field [3] and has promising clinical applications in hemodynamics monitoring, brain, burns and et al. However, One important issue in SFDI is the imaging speed and motion artefacts [4]. In order to decouple scattering from absorption, the measurement of the modulation transfer function (MTF) of the turbid medium at no less than two modulation frequencies is required. The standard three phase demodulation method requires three images at different phase delay $(0,2 \pi / 3$, and $4 \pi / 3)$ to compute MTF at one spatial frequency (one AC component), which in turn hinders SFDI in real time applications such as when monitoring fast temporal dynamics.

Recently, several fast demodulation methods have been proposed to improve upon the standard 3-phase demodulation approach for SFDI. The single snapshot optical properties method (SSOP) developed by Vervandier et al [5] uses a line by line Fourier transform of an SFDI intensity image and processes in the frequency space to extract one DC and one AC image from a single frame of data to recover the optical property. An alternative approach proposed by Nadeau et al [6] uses a 2-D Hilbert transform by employing a complex-valued spiral phase function in the Fourier space. The Hilbert demodulation method requires one single frame of image to recover one AC component at the specific frequency. We also have presented a novel single snapshot multiple frequency demodulation (SSMD) method to extract one DC and multiple AC components simultaneously from a single structured-illuminated image at once using the orthogonality of harmonic functions [7]. The multiple AC components can have arbitrary orientations, amplitudes, and modulation frequencies subject to the limitation of the implementation device, offering the design freedom to achieve the best signal to noise performance over the medium response at all the modulation frequencies of interest. The performance of SSMD is demonstrated with experiments on both tissue mimicking phantoms and in vivo for recovering optical properties by comparing with the standard three-phase approach. SSMD not only is much faster and reduces motion artefacts but also exhibits excellent noise suppression in imaging as well. The application of SSMD may open up SFDI for real time mapping of the optical properties of a dynamic turbid medium and monitoring of fast temporal evolutions.

Optical Tomography and Spectroscopy of Tissue XII, edited by Bruce J. Tromberg, Arjun G. Yodh,

Eva Marie Sevick-Muraca, Robert R. Alfano, Proc. of SPIE Vol. 10059, 100590Z · @ 2017 SPIE

CCC code: $1605-7422 / 17 / \$ 18 \cdot$ doi: $10.1117 / 12.2253992$ 


\section{METHODOLOGY}

\subsection{Spatial frequency domain imaging}

SFDI maps the optical properties of a turbid medium from its reflectance images under structured illuminations. Assuming an incident intensity pattern of $I_{0}=I_{D C}^{(0)}+I_{A C}^{(0)} \cos \left(2 \pi f_{x} x+2 \pi f_{y} y+\phi\right)$ in SFDI, the backscattering light intensity can be described as:

$$
I(x, y)=I_{D C}(x, y)+I_{A C}(x, y) \cos \left(2 \pi f_{x} x+2 \pi f_{y} y+\phi\right) .
$$

where $f_{\mathrm{x}}, f_{\mathrm{y}}$ are the modulating spatial frequencies along the $x$ and $y$ directions, respectively, and $\phi$ is the phase. The demodulation is to extract the $\mathrm{AC}$ amplitude $I_{A C}(x, y)$ from $I(x, y)$. The ratio $I_{A C} / I_{A C}^{(0)}$ represents the modulation transfer function (MTF) of the turbid medium at the spatial frequency $\left(f_{x}, f_{y}\right)$ after removing the imaging system response characteristics through calibration. In the standard three-phase demodulation, three images are acquired at three equally separated phases $\phi_{1,2,3}=(0,2 \pi / 3,4 \pi / 3)$ for each $\mathrm{AC}$ spatial frequency. The $\mathrm{AC}$ amplitude $I_{A C}(x, y)$ is solved pixel-by-pixel as

$$
I_{A C}(x, y)=\frac{\sqrt{2}}{3}\left[\left(I_{1}-I_{2}\right)^{2}+\left(I_{2}-I_{3}\right)^{2}+\left(I_{3}-I_{1}\right)^{2}\right]^{\frac{1}{2}} .
$$

where $I_{1,2,3}$ is the backscattered light intensity corresponding to the phases $\phi_{1,2,3}$, respectively. Once the MTF of the turbid medium at two or more modulation frequencies is obtained, the optical properties of the medium are then determined by comparing to the light transport model such as radiative transfer or diffusion in the Fourier domain [8-10].

\subsection{Single snapshot multiple frequency demodulation technique}

Here we briefly outline the key ideas of single snapshot multiple frequency demodulation technique and the details have been presented in [7]. The main power of structured illumination with digital micromirror devices (DMDs) is that the pattern of the incident light on the surface of a specimen can be freely controlled and altered with precise knowledge. SSMD adopts a structured illumination consisting of multiple harmonics simultaneously. The light pattern on the surface of the specimen and the backscattered light intensity is given, respectively, by:

$$
\begin{gathered}
I_{0}(x, y)=I_{D C}^{(0)}+\sum_{i=1}^{k} I_{A C, i}^{(0)} \cos \left[2 \pi\left(f_{x, i} x+f_{\mathrm{y}, i} y\right)+\phi_{i}\right] . \\
I(x, y)=I_{D C}(x, y)+\sum_{i=1}^{k} I_{A C, i}(x, y) \cos \left[2 \pi\left(f_{x, i} x+f_{\mathrm{y}, i} y\right)+\phi_{i}\right] .
\end{gathered}
$$

where $k \geq 1$ is the number of AC components, $I_{A C, i}^{(0)}$ and $I_{A C, i}$ are, respectively, the incident and backscattering amplitudes of the $i$-th AC component modulated at the spatial frequency $\left(f_{x, i}, f_{y, i}\right)$ and of phase $\phi_{i}$. Note that the spatial modulation frequencies can be prescribed exactly by DMD (subject to the limitation of DMD imposed by its the pixelated nature and size) once the SFDI system has been setup and calibrated.

The key in SSMD is that the $k$ different spatial frequencies are chosen to take the form of $f_{\mathrm{x}, i}=m_{i} / T_{1}$ and $f_{y, i}=n_{i} / T_{2}$ where $-T_{1}<m_{i}<T_{1},-T_{2}<n_{i}<T_{2}$, and $m_{i}, n_{i}, T_{1}$, and $T_{2}$ are all integers. In other words, $T_{1}$ and $T_{2}$ define the smallest common multiples of all the AC modulating spatial periods along the horizontal and vertical directions, respectively. As in the standard three-phase demodulation method, $I_{A C, i}(x, y)$ is further assumed to be slow varying and can be treated as a constant over the sliding window of size $T_{1} \times T_{2}$ pixels. Each AC component $I_{A C, i}$ is given by

$$
\begin{gathered}
I_{A C, i}=\frac{\sqrt{\left[\iint_{\sigma} I(x, y) \cos \left(2 \pi f_{x, i} x+2 \pi f_{\mathrm{y}, i} y\right) d x d y\right]^{2}+\left[\iint_{\sigma} I(x, y) \sin \left(2 \pi f_{x, i} x+2 \pi f_{\mathrm{y}, i} y\right) d x d y\right]^{2}}}{\iint_{\cos ^{2}\left(2 \pi f_{x, i} x+2 \pi f_{\mathrm{y}, i} y\right) d x d y}} . \\
I_{D C}=\frac{1}{T_{1} \times T_{2}} \iint_{\sigma} I(x, y) d x d y .
\end{gathered}
$$


where the integration is performed over the sliding window $\sigma$. Here, in order to remove the requirement of the knowledge of the phase $\phi_{i}$, a sinusoid and cosine kernel with the same spatial modulation frequency $\left(f_{x, i}, f_{y, i}\right)$ and of size $T_{1} \times T_{2}$, respectively, is applied to filter $I(x, y)$, and the two resulting images are then combined to recover the corresponding AC component.

A schematic diagram of the SSMD is illustrated in FIG.1. As one example, the superimposed image consists of one uniform DC background, a “中” AC image and a “华” AC image, where “中” is modulated horizontally at the spatial frequency $f_{1}$ and “华” is modulated along the $45^{\circ}$ direction at the spatial frequency $f_{2}$. First, we determine the sliding window (kernel) size $T_{1} \times T_{2}$ that $T_{1}$ equals the least common multiple of $1 / f_{1}$ and $\sqrt{2} / f_{2}$, and $T_{2}$ equals $\sqrt{2} / f_{2}$. A sinusoid and cosine kernel of size $T_{1} \times T_{2}$, respectively, is then applied to filter the superimposed image and the two resulting images are combined to recover the corresponding AC component according to Eq. (5). The DC image is extracted by a direct averaging over the $T_{1} \times T_{2}$ window (i.e., a unity kernel). Each individual image of “中” and “华” is then demodulated with high fidelity (the rightmost column of FIG. 1).

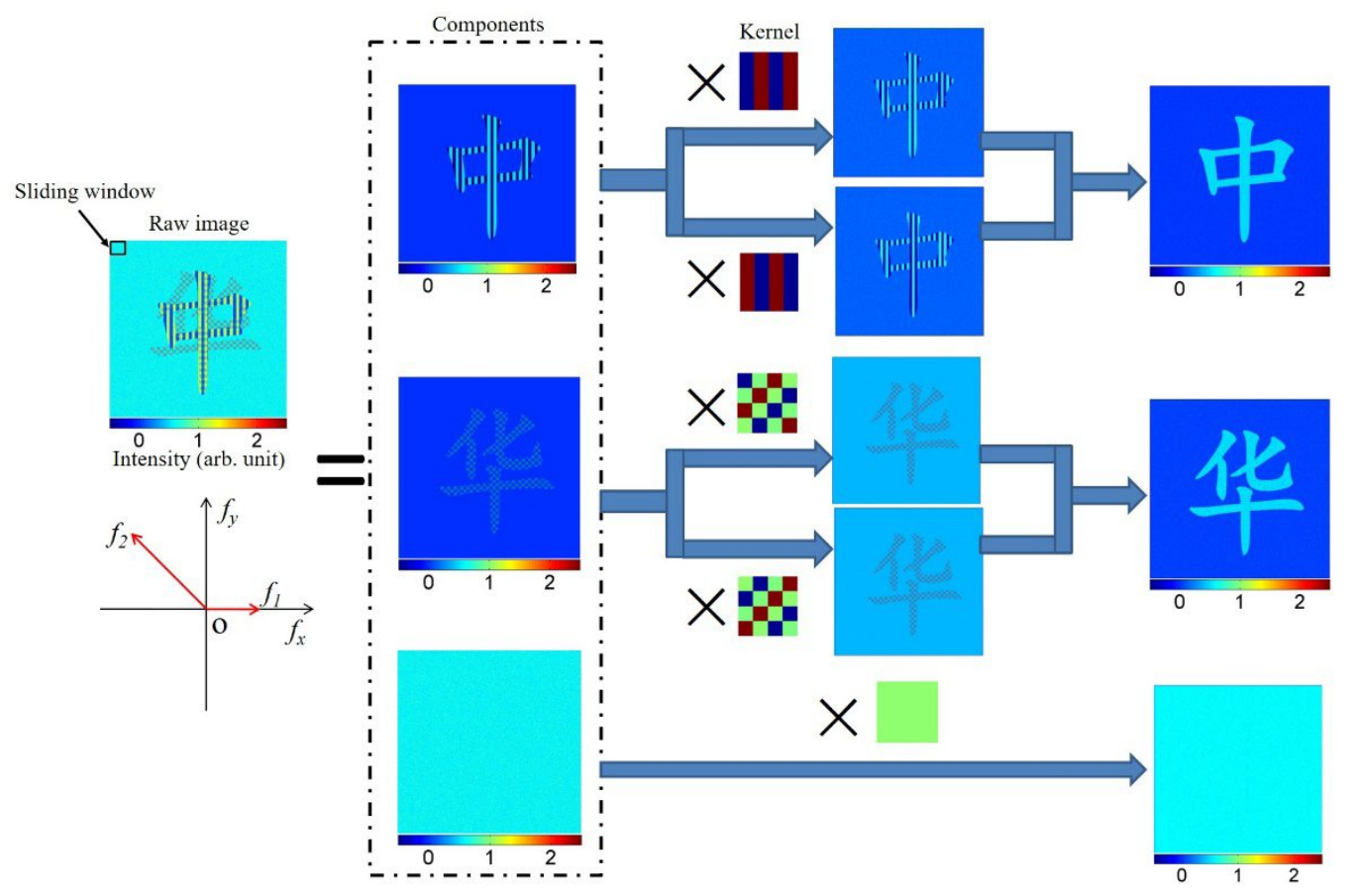

FIG. 1. The schematic diagram of single snapshot multiple frequency demodulation method. The single snapshot displayed in the leftmost column is the image recorded, for example, by a CCD camera from structured illumination for the synthesized sample. Here this image consists of one uniform DC background, a “中” AC image modulated horizontally with the spatial frequency $f_{1}$, and a “华” AC image modulated diagonally with the spatial frequency $f_{2}$. The modulating spatial frequencies $f_{1}$ and $f_{2}$ are displayed in the phasor diagram below the single snapshot. For each component of known frequency, a sinusoid and cosine kernel of size $T_{1} \times T_{2}$, respectively, is applied to filter the single snapshot and the two resulting images are combined to recover the corresponding AC component according to Eq. (5). The DC image is extracted by a direct averaging over the $T_{1} \times T_{2}$ window.

\section{RESULTS AND DISCUSSION}

\subsection{Homogenous phantom experiment}

The 2D maps of the optical properties of the Intralipid-2\% suspension reconstructed by the three-phase method and SSMD are then compared in FIG. 2. The incident beam is spatially modulated at $f_{1}=0.487 \mathrm{~mm}^{-1}$ vertically and $/$ or $f_{2}=$ 
$0.689 \mathrm{~mm}^{-1}$ diagonally. The optical properties of the medium are reconstructed by a look-up table based on the enhanced diffusion approximation [11]. The three-phase method is applied to measurements modulated at one single frequency alone and SSMD to measurements modulated at either single or two frequencies simultaneously. Both methods recover the optical properties of the Intralipid- $2 \%$ well with the absorption and reduced scattering coefficients of $3.10 \times 10^{-4} \mathrm{~mm}^{-1}$ and $2.63 \mathrm{~mm}^{-1}$ from $f_{1}$ demodulation and $3.69 \times 10^{-4} \mathrm{~mm}^{-1}$ and $3.14 \mathrm{~mm}^{-1}$ from $f_{2}$ demodulation, respectively. The same suspension was measured by an oblique incidence diffuse reflectance method [12] which yields $\mu_{\mathrm{a}}=3.0 \times 10^{-4} \mathrm{~mm}^{-1}$ and $\mu_{s}^{\prime}=2.60 \mathrm{~mm}^{-1}$. The recovered $\mu_{\mathrm{a}}$ and $\mu_{s}^{\prime}$ at $f_{2}$ are slightly overestimated compared to the expected values partly attributed to the gradual loss of the accuracy of the diffusion approximation at higher modulation frequencies [13-15]. The optical property maps produced with SSMD overall show much less fluctuations comparing to those with the standard three-phase method. The performance of SSMD also holds up well when modulating at either single or multiple frequencies simultaneously. Note the intensity of the reflectance inside the central region is stronger than the surrounding region owing to the boundary effects. The boundary effects also show up clearly in the recovered optical properties maps, in particular, nearby the left edge in the $f_{1}$ case with SSMD.
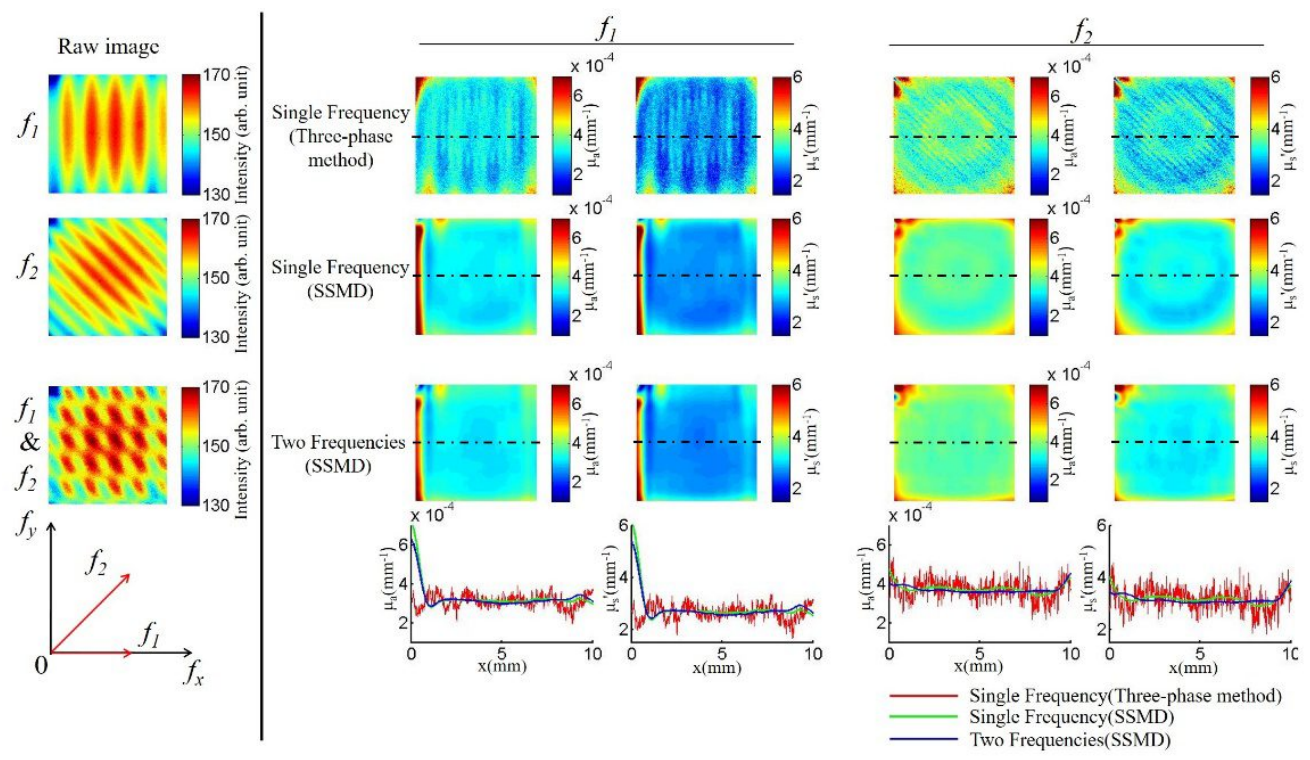

(a)

(b)

FIG. 2. Comparison of recovered optical property maps by the standard three-phase method and SSMD from measurements on Intralipid- $2 \%$ suspension. (a) Raw intensity images measured when modulating at $f_{1}=0.487 \mathrm{~mm}^{-1}, f_{2}=0.689 \mathrm{~mm}^{-1}$, and $f_{1}$ $\& f_{2}$ simultaneously. (b) Recovered optical property maps from the three-phase method (modulated at one single frequency alone), SSMD (modulated at one single frequency), and SSMD (modulated at both frequencies simultaneously).

\subsection{In vivo forearms experiment}

Lastly, forearms of healthy young adults were imaged by the real time SSMD-SFDI system at two wavelengths $\lambda=$ $623 \mathrm{~nm}$ (Red) and $540 \mathrm{~nm}$ (Green). The spatial modulation frequency is $f=0.2 \mathrm{~mm}^{-1}$. Five images were recorded per second by a digital color camera (Point Grey Grasshop3 GS3-U3-51S5C) under paced breathing at frequencies of 0.0833 $\mathrm{s}^{-1}$. The optical parameters $\left(\mu_{\mathrm{a}}\right.$ and $\left.\mu_{s}^{\prime}\right)$ are calculated from each image based on the diffusion approximation and the SSMD method. We first performed Fourier analysis of the time traces of the absorption coefficient and only retained those experiments in which the power spectra of $\mu_{\mathrm{a}}$ showed a peak at the frequency of paced breathing (see FIG. 3.(ac)). We removed temporal drifts from $\mu_{\mathrm{a}}$ by a third-order polynomial detrending. The detrended time traces of $\mu_{\mathrm{a}}$ were then filtered using a linear-phase band-pass finite impulse response filter based on the Parks-McClellan algorithm (see FIG. 3.(d, e)). The coherent hemodynamics signal due to paced breathing was clearly recovered with our SSMD-SFDI system $[16,17]$. 

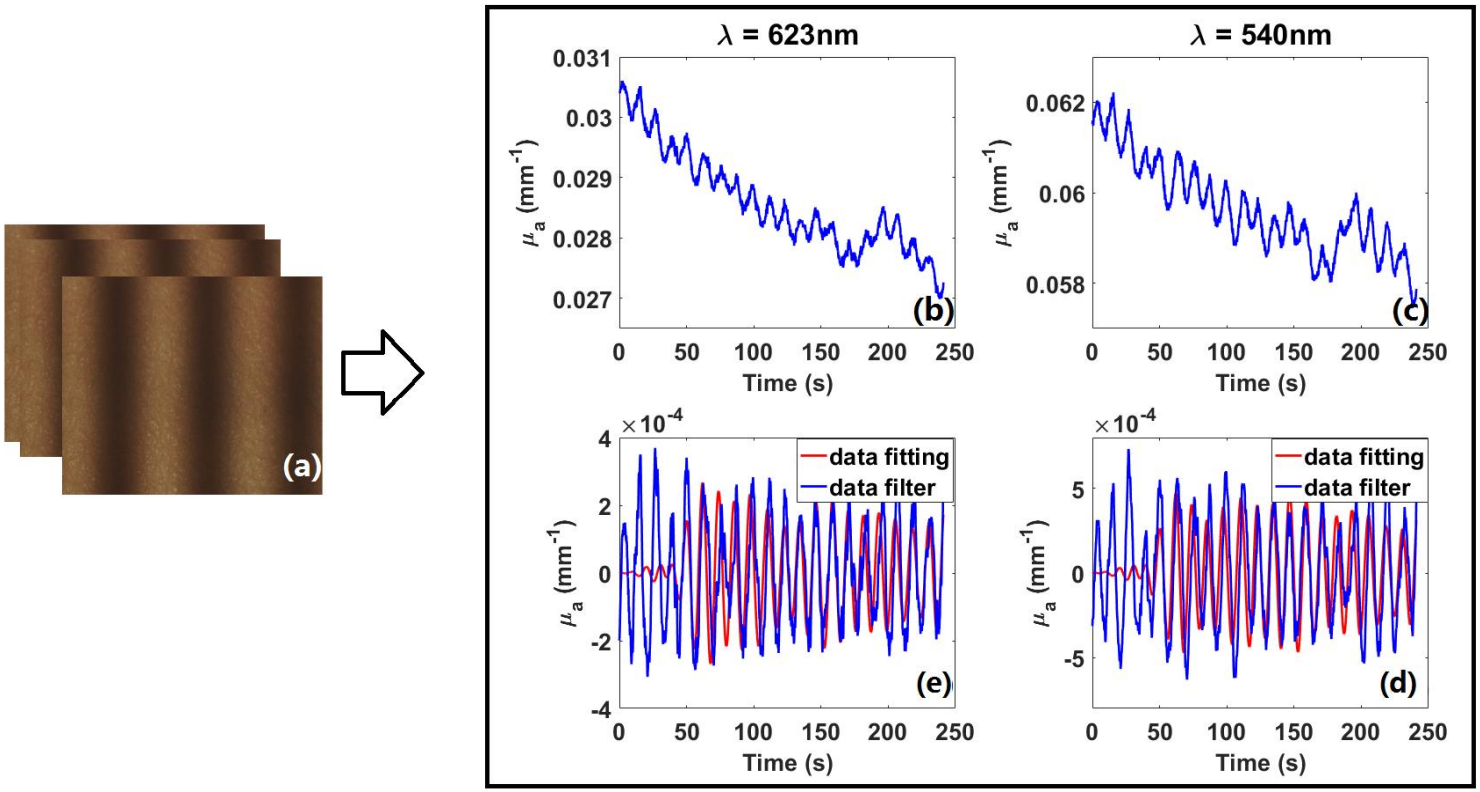

FIG. 3. Acquisition of dynamic optical parameters $\left(\mu_{\mathrm{a}}\right)$. (a) Raw images of the forearm recorded in the SFDI system. (b), (c) The extracted absorption coefficient in Red and Green channels. (d), (e)The filtered absorption coefficient after removing temporal drifts and filtered using a linear-phase band-pass finite impulse response filter based on the ParksMcClellan algorithm. The first 100s data was discarded.

\section{CONCLUSION}

We have presented a novel Single Snapshot Multiple frequency Demodulation (SSMD) method enabling single snapshot wide field imaging of optical properties of turbid media in the Spatial Frequency Domain. SSMD makes use of the orthogonality of harmonic functions and extracts the modulation transfer function (MTF) at multiple modulation frequencies and of arbitrary orientations and amplitudes subject to the implementation device simultaneously from a single structured-illuminated image at once. The excellent performance of SSMD has been demonstrated with experiment on both tissue mimicking phantoms and in vivo for recovering optical properties by comparing to the conventional three-phase approach. SSMD not only increases significantly the data acquisition speed and reduces motion artefacts but also exhibits excellent noise suppression in imaging as well. SSMD is ideal in the implementation of a realtime spatial frequency domain imaging platform, which will open up SFDI for vast applications in, for example, mapping the optical properties of a dynamic turbid medium and monitoring fast temporal evolutions.

\section{ACKNOWLEDGMENTS}

This work is supported by National Nature Science Foundation of China (81470081), Key Research Program of Zhejiang Natural Science Foundation of China (Z16H180005), Fundamental Research Funds of Wenzhou Medical University (89213018), and Zhejiang Xinmiao Talents Program (2015R413073).

\section{REFERENCES}

[1] S. D. Konecky, A. Mazhar, D. Cuccia et al., "Quantitative optical tomography of sub-surface heterogeneities using spatially modulated structured light," Optics Express, 17(17), 14780-14790 (2009).

[2] T. D. O'Sullivan, A. E. Cerussi, D. J. Cuccia et al., "Diffuse optical imaging using spatially and temporally modulated light," Journal of Biomedical Optics, 17(7), (2012).

[3] S. C. Kanick, D. M. McClatchy, V. Krishnaswamy et al., "Sub-diffusive scattering parameter maps recovered using wide-field high-frequency structured light imaging," Biomedical Optics Express, 5(10), 3376-3390 (2014).

[4] J. Q. Nguyen, R. B. Saager, D. J. Cuccia et al., "Effects of motion on optical properties in the spatial frequency domain," Journal of Biomedical Optics, 16(12), (2011). 
[5] J. Vervandier, and S. Gioux, "Single snapshot imaging of optical properties," Biomed Opt Express, 4(12), 2938-44 (2013).

[6] K. P. Nadeau, A. J. Durkin, and B. J. Tromberg, "Advanced demodulation technique for the extraction of tissue optical properties and structural orientation contrast in the spatial frequency domain," J Biomed Opt, 19(5), 056013 (2014).

[7] M. Xu, Z. Cao, W. Lin et al., "Single snapshot multiple frequency modulated imaging of subsurface optical properties of turbid media with structured light," AIP Advances, 6, (2016).

[8] D. J. Cuccia, F. Bevilacqua, A. J. Durkin et al., "Quantitation and mapping of tissue optical properties using modulated imaging," Journal of Biomedical Optics, 14(2), (2009).

[9] T. A. Erickson, A. Mazhar, D. Cuccia et al., "Lookup-table method for imaging optical properties with structured illumination beyond the diffusion theory regime," J Biomed Opt, 15(3), 036013 (2010).

[10] M. Xu, W. Cai, M. Lax et al., "Photon-transport forward model for imaging in turbid media," Optics Letters, 26(14), 1066-1068 (2001).

[11] M. Xu, "Low-coherence enhanced backscattering beyond diffusion," Opt Lett, 33(11), 1246-8 (2008).

[12] M. Xu, M. Alrubaiee, S. Gayen et al., "Determination of light absorption, scattering and anisotropy factor of a highly scattering medium using backscattered circularly polarized light." Biomedical Optics (BiOS) , 64350J-64350J-6 (2007).

[13] M. Xu, "Diagnosis of the phase function of random media from light reflectance," Sci Rep, 6, 22535 (2016).

[14] M. Reilly, and M. Xu, "Analytical model for sub-diffusive light reflection and the application to spatial frequencydomain imaging." SPIE BiOS, 93191A-93191A-6 (2015).

[15] W. Lin, Z. Cao, B. Zeng et al., "Quantitative modulated imaging of turbid media in the high spatial frequency domain." SPIE BiOS, 97010Y-97010Y-7 (2016).

[16] S. Fantini, "Dynamic model for the tissue concentration and oxygen saturation of hemoglobin in relation to blood volume, flow velocity, and oxygen consumption: Implications for functional neuroimaging and coherent hemodynamics spectroscopy (CHS)," Neuroimage, 85(2), 202-221 (2013).

[17] X. Chen, Z. Cao, W. Lin et al., "Microcirculation monitoring with real time spatial frequency domain imaging." International Conference on Innovative Optical Health Science, 102450J-102450J-6 (2017). 